\title{
Joining of Melt-Textured YBCO: a Direct Contact Method
}

\author{
Lihua Chen $^{\mathrm{a}}$, H. Claus ${ }^{\mathrm{a}, \mathrm{b}}$, A. P. Paulikas ${ }^{\mathrm{a}}$, Hong Zheng ${ }^{\mathrm{a}}$, and B. W. Veal ${ }^{\mathrm{a}}$ \\ ${ }^{\mathrm{a}}$ Materials Science Division, Argonne National Laboratory, Argonne Illinois 60439 \\ ${ }^{b}$ Department of Physics, University of Illinois at Chicago, Chicago IL 60607-7059
}

\begin{abstract}
We report a method for making weld joints, capable of transmitting high supercurrent densities, in bulk samples of melt textured YBCO. The joining procedure is carried out in a flowing atmosphere of $\mathrm{O}_{2}$ to eliminate problems associated with nitrogen gas, which can become trapped in the joint. No filler or fluxing material is used. The method can be used to join large areas (several $\mathrm{cm}^{2}$ ) that are capable of transmitting supercurrent densities exceeding $10^{4} \mathrm{~A} / \mathrm{cm}^{2}$.
\end{abstract}




\section{Introduction}

Bulk textured superconductors continue to hold promise for important new technological applications including high power density motors/generators, levitators, and fault current limiters. However, the bulk HTS materials cannot generally be directly produced in the melt-texturing process in sizes that are sufficiently large for these applications. Thus, methods are needed to join monoliths, of a size that can be readily and inexpensively fabricated, into final assemblies of desired size and shape for a given application. Of course, current densities across the joints must be comparable to those of the surrounding bulk material.

A number of approaches have been used, with varying degrees of success, to obtain joints capable of transporting supercurrents with high critical current densities. These approaches can be divided into two general categories: (1) joining with the use of a high $\mathrm{T}_{\mathrm{c}}$ filler material that has a decomposition temperature lower than that of the parts to be joined, and (2) direct contact joining which might include pressure loading (weighting) of the joint. For joining YBCO, fillers include YbBCO [1-3], TmBCO [4] and ErBCO [5]. Recently, successful joints were made using Ag-doped YBCO as the filler agent [6,7]. Direct contact welds, processed in air, have been successfully demonstrated for small area joints (several square $\mathrm{mm}$ of area) [8-10].

\section{Procedure and Tests}

For our joining procedure, monoliths are stacked as shown in Fig. 1 so that $a-b$ plane directions are coincident and c-axis directions are parallel. For development 
of the joining procedure, square monoliths were cut and rejoined. The diamondsaw cut surfaces were cleaned ultrasonically and arranged as shown in Fig. 1 so that one half of the sample ( 15 - $20 \mathrm{~g}$ total) weights the joint during the processing step. Then the sample was processed according to the time-temperature schedule shown in Fig. 1. This processing step was carried out in flowing $\mathrm{O}_{2}$ at ambient pressure.

The use of an oxygen atmosphere during the joining step appears to be a critical feature for making successful large area weld joints. At the high processing temperature $\left(\sim 1000^{\circ} \mathrm{C}\right)$, entrapped oxygen will diffuse rapidly from the vicinity of the joint. However, if the processing is carried out in air $\left(\sim 80 \% \mathrm{~N}_{2}, 20 \% \mathrm{O}_{2}\right)$, a significant amount of nitrogen gas becomes trapped in the joint. Apparently, this trapped gas severely limits the quality of the joint, especially for large areas. It has been previously reported that melt textured bulks can be produced void-free and nearly $100 \%$ dense by including a processing step in $\mathrm{O}_{2}[7,11]$.

After the samples have been joined, the assembly is treated in flowing oxygen for 7 to 10 days at $450^{\circ} \mathrm{C}$ to fix the oxygen stoichiometry. Fig. 2 shows trapped field measurements from a square welded monolith, taken to test the quality of the joint. Results are shown for a slice of the sample, $16 \mathrm{~mm}$ on a side and $3 \mathrm{~mm}$ thick that was cut across the joint, perpendicular to the c-axis. The sample was cooled to 77 $\mathrm{K}$ in a field of $5 \mathrm{kG}$, the field was turned off, and the trapped field was scanned. Note that the trapped field is symmetrically distributed, with no degradation in the vicinity of the weld joint (at the center of the sample). A Jc measurement from a sample cut across the joint yields $1.04 \times 10^{4} \mathrm{~A} / \mathrm{cm}^{2}$, a value that is comparable to the bulk Jc. For comparison, we also show, in Fig. 3, the trapped field distribution from a sample of comparable dimensions, but containing an unsuccessful weld 
joint. In this case, the field is profoundly reduced at the sample center, in the vicinity of the joint.

Welds, such as that shown in Fig. 2, involving the rejoining of a cut monolith, are needed for developing the joining process. Rejoining of such faces avoids problems of crystallographic alignment. However, for applications purposes, it will be necessary to join independently prepared monoliths, where is becomes necessary to establish and maintain bi-axial alignment across the joined interface. Fig. 4 shows an example of joined, independently prepared monoliths. As Fig. 4 indicates, there is substantial supercurrent flow across the weld-joint, albeit reduced from the bulk value as indicated by the reduction in the trapped field in the region of the joint. Note, however, that the failed joint in Fig 3 shows a greater depression of the trapped field in the vicinity of the joint.

Fig. 4 also shows trapped field measurements from a ring, which was core-drilled from the center of the sample, across the weld. The ring geometry allows easy determination of the critical current density across the joint from the magnitude of the magnetic field trapped in the bore of the ring [7]. The ring (14.9 mm OD, 6 $\mathrm{mm}$ ID, and $3.4 \mathrm{~mm}$ thick) trapped a field of $0.75 \mathrm{kG}$ on the ring axis (Fig. 4b). The trapped field is sustained by a 620 A circulating current, yielding a Jc of 4000 $\mathrm{A} / \mathrm{cm}^{2}$, about one third of the bulk Jc. Presumably this degradation, relative to the bulk, results from inadequate grain alignment (cut samples, which preserve crystallographic alignment, were successfully rejoined with high reliability). According to ref 7 (see Fig. 10 of ref. 7), this $\mathbf{J}_{c}$ corresponds to a misorientation angle of approximately $8^{\circ}$, assuming that the miscut is an [001] tilt misorienation. Nonetheless, it appears promising that a simple procedure can be developed for aligning and joining independently prepared monoliths. 
A simple procedure was used to align the monoliths for welding. A thin layer was cleaved from the top surface of each piece, in the vicinity of the joining faces. These cleaved surfaces were used to establish c-axis alignment. a-b plane directions were identified from the orientation of the seed crystal or from the square patterns in the mosaic growth structure, visible on the cleaved surfaces.

In addition to joining, the thermal processing procedure in $\mathrm{O}_{2}$ can be used to repair cracks in textured monoliths. Fig. 5 (panel a) shows the trapped field in a ring sample (27 mm OD, $11 \mathrm{~mm}$ ID, $6 \mathrm{~mm}$ thick) which contained a crack, or cracks, preventing the circumferential flow of persistent current. The trapped field is associated only with the bulk material; essentially no field is generated at the ring center from a circulating current. After thermal processing, using the same schedule as shown in Fig. 1, the $\operatorname{crack}(\mathrm{s})$ are healed and no weak link behavior is indicated by the trapped field measurement. A circulating current of $2500 \mathrm{amps}$ generates a field of about $1.7 \mathrm{kG}$ at the ring center corresponding to a $\mathrm{J}_{\mathrm{c}}$ of 5000 $\mathrm{A} / \mathrm{cm}^{2}$.

Summary

We report a direct contact procedure for joining bulk YBCO monoliths to obtain a high $-J_{c}$ superconducting weld joint. The processing is carried out in an atmosphere of flowing $\mathrm{O}_{2}$ to avoid the deleterious effect of trapped nitrogen gas within the joint. Large area welds $\left(\right.$ several $\mathrm{cm}^{2}$ ) with critical current densities exceeding $10^{4} \mathrm{~A} / \mathrm{cm}^{2}$ have been successfully demonstrated. The procedure can also be used to seal cracks that develop in monoliths. 


\section{Acknowledgements}

This work was supported by the U.S. Department of Energy, Basic Energy Sciences-Materials Sciences-Metals, Ceramic and Engineering Sciences, under contract W-31-109-ENG-38. 


\section{References}

[1] K. Kimura, K. Miyamoto, M. Hashimoto, Advances in Superconductivity VII, Springer Verlag (Tokyo), 681 (1995).

[2] M. P. Delmare, H. Walter, B. Brigmann, A. Leeders, H. C. Freyhardt, Physica C329, 257 (2000).

[3] J. G. Noudem, E. S. Reddy, M. Tarka, M. Noe and G. J. Schmitz, Supercond. Sci. Technol. 14, 363 (2001).

[4] H. Zheng, M. Jiang, R. Nikolova, U. Welp, A. P. Paulikas, Yi Huang, G. W. Crabtree, B. W. Veal, Physica C 322, 1, (1999).

[5] S. J. Manton, C. Beduz and Y. Yang, IEEE Transactions on Applied Superconductivity, Vol. 9, p. 2089 (1999).

[6] T. Puig, P. Rodrigues Jr, A. E. Carillo, X. Obradors, H. Zheng, U. Welp, L. Chen, H. Claus, B. W. Veal and G. W. Crabtree, Physica C (in press).

[7] H. Claus, U. Welp, H. Zheng, L. Chen, A. P. Paulikas, B. W. Veal, K. E. Gray, G. W. Crabtree, Phys. Rev. B (in press).

[8] K. Salama, V. Selvamanickan, Appl. Phys. Lett. 60, 898 (1992).

[9] W. Lo, D. A. Cardwell, A. D. Bradley, R. A. Doyle, Y. H. Shi, S. Loyd, IEEE Trans, on Applied Superconductivity 9, 2042 (1999).

[10] D. Shi, Appl. Phys. Lett. 66, 2573 (1995).

[11] N. Sakai, D. Ishihara, K. Inoue, K. Ogasawara and M. Murakami, The 2000 International Workshop on Superconductivity, June 19-22, 2000, Matsue-shi, Shimane, Japan, p.167. 
Figure Captions

Fig. 1. Panel (a): For joining, bulk monoliths are positioned with their c-axes parallel and with a,b plane directions aligned. The joint is weighted by the upper half of the sample. Panel (b): Thermal processing schedule, in $\mathrm{O}_{2}$, for making the direct contact welds. $\mathrm{T}_{\mathrm{D}}=1025^{\circ} \mathrm{C}$ is the decomposition temperature of $\mathrm{YBCO}$.

Fig. 2. A scanning Hall probe measurement of the trapped field in a square monolith that was measured after it was cut into two approximately equal pieces and rejoined. The field profile shown is that of a slice containing the weld joint through its center. The profile gives no indication of weak link behavior at the weld joint.

Fig. 3. The trapped field pattern in a bisected square monolith that was obtained after an unsuccessful weld. The field minimum at the cut is determined by the resolution of the scanning Hall probe.

Fig. 4. Panel (a): The trapped field profile in a sample assembled from two independently grown monoliths that were aligned and welded. Panel; (b): The trapped field profile in a ring cut across the weld joint. A reduced current density $\left(4000 \mathrm{~A} / \mathrm{cm}^{2}\right.$, see text) is observed at the joint.

Fig. 5. Panel (a): The trapped field in a ring sample containing a crack (or cracks) that prevent the flow of circulating persistent current. Essentially no field is generated at the ring center. Panel (b): The trapped field after the ring was repaired using the described welding procedure. Note the substantial field at the ring center that is now generated by a circulating current. 

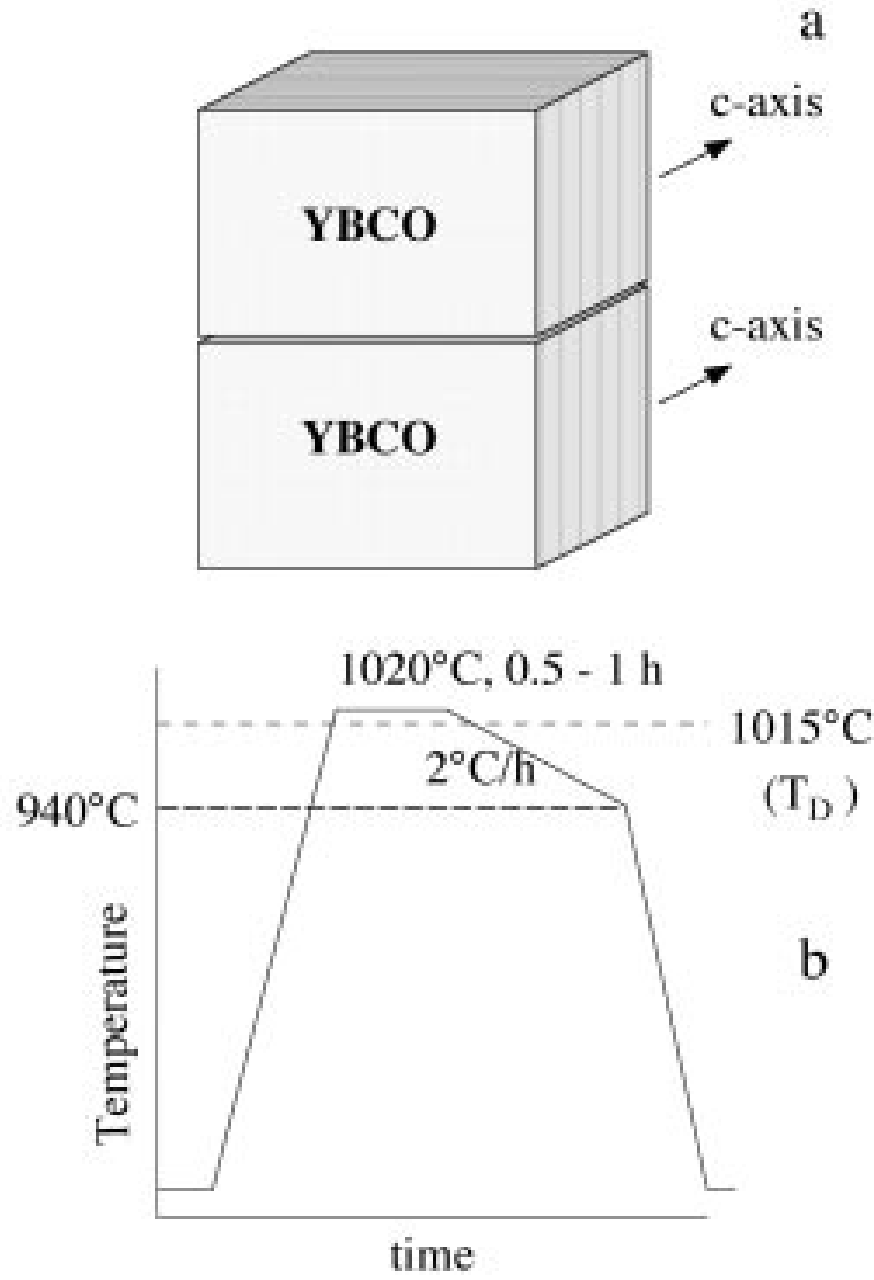

Fig. 1

L. Chen et al. 


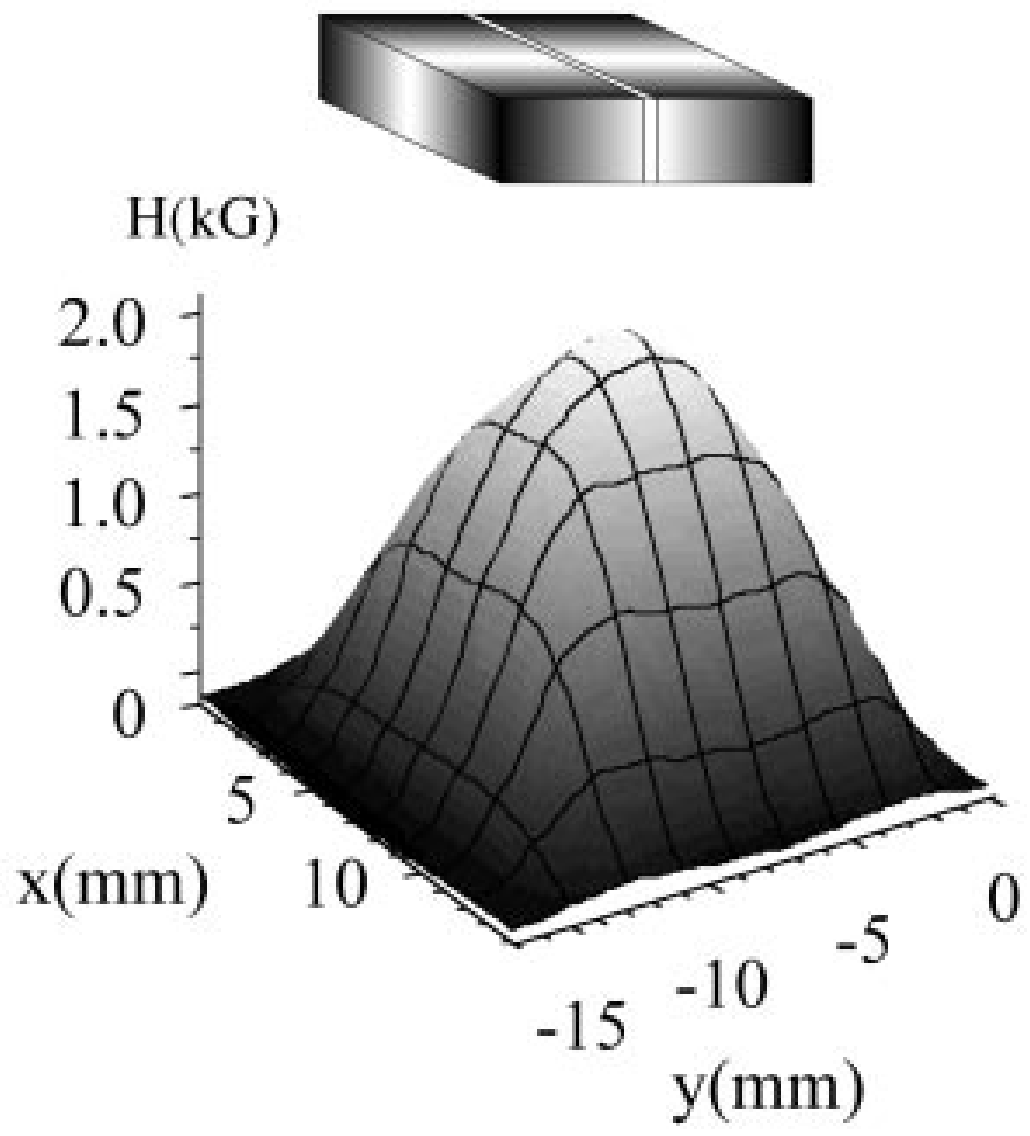

Fig. 2

L. Chen et al. 


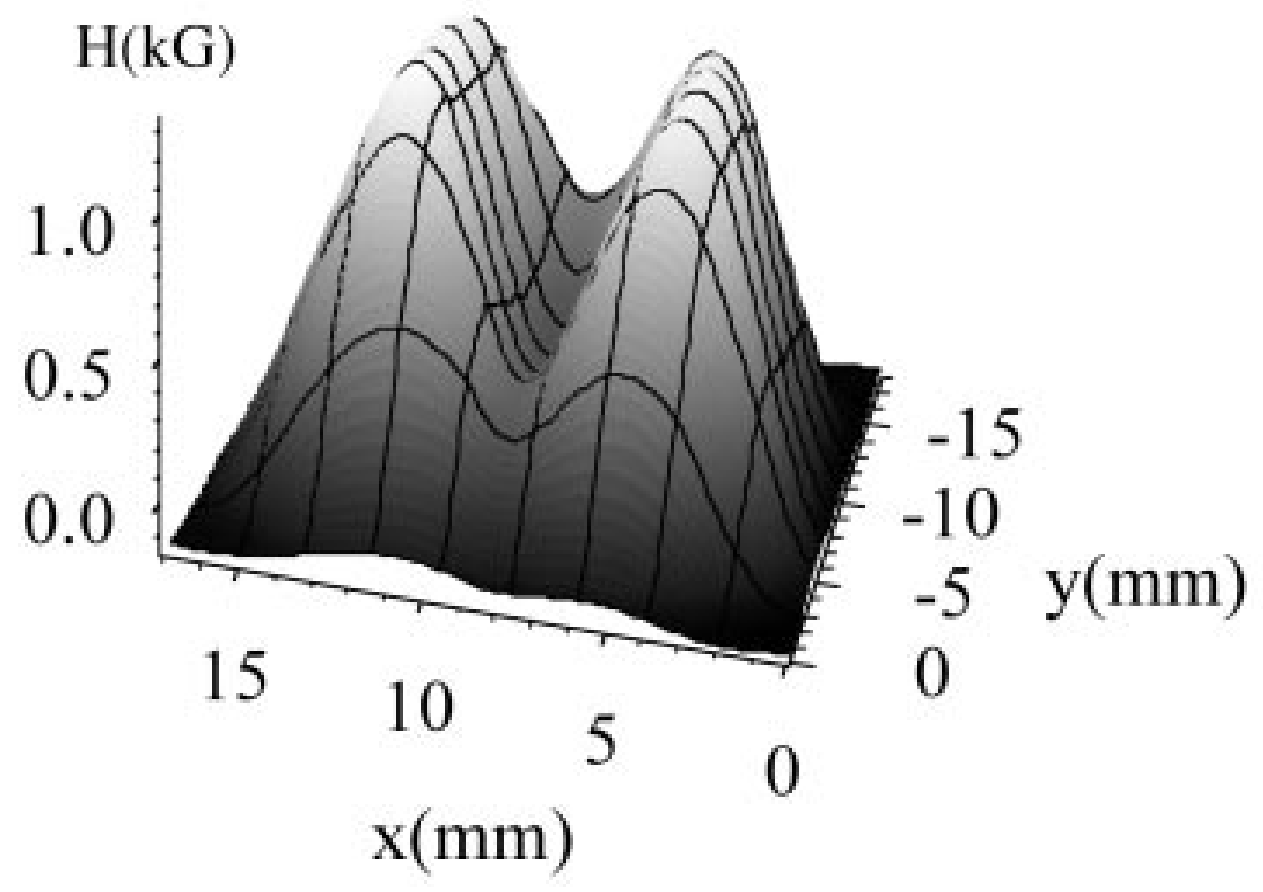

Fig. 3

L. Chen et al. 

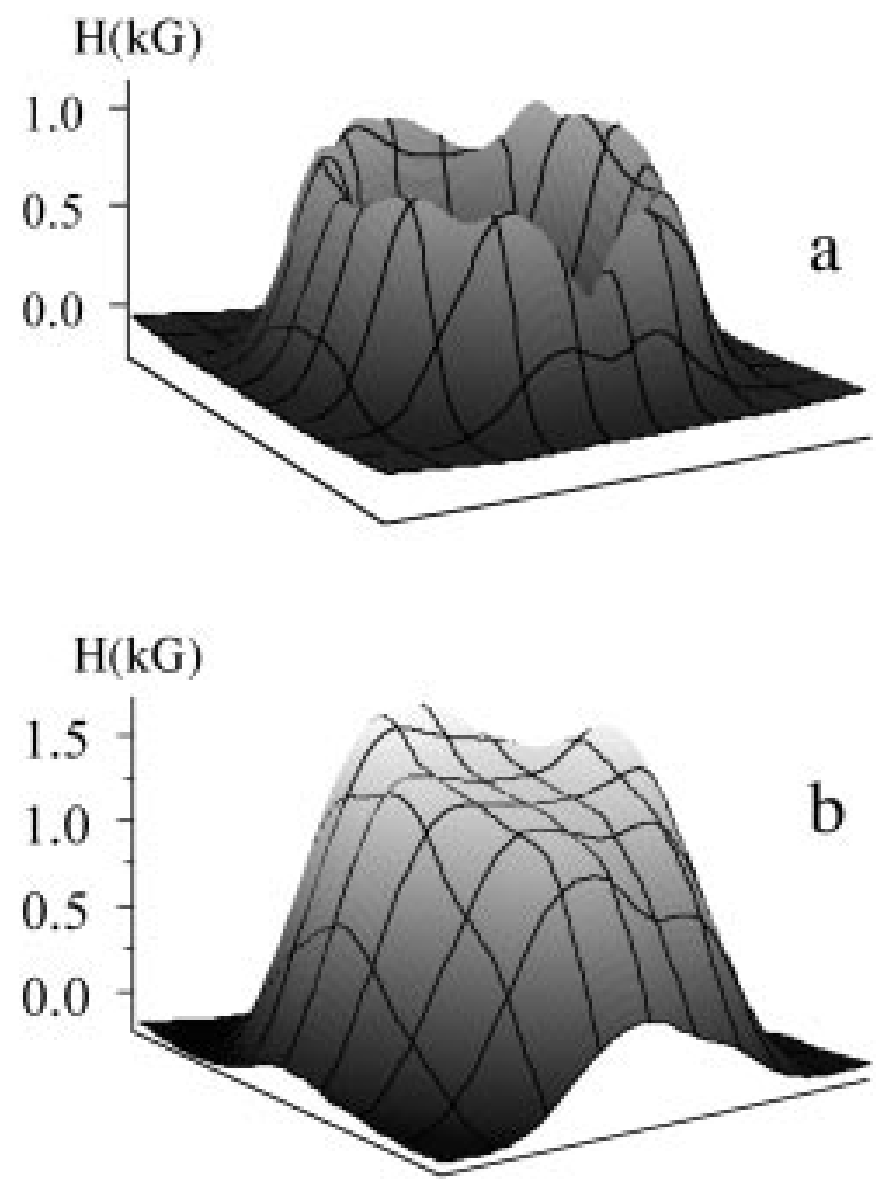

Fig. 5

L. Chen et al. 

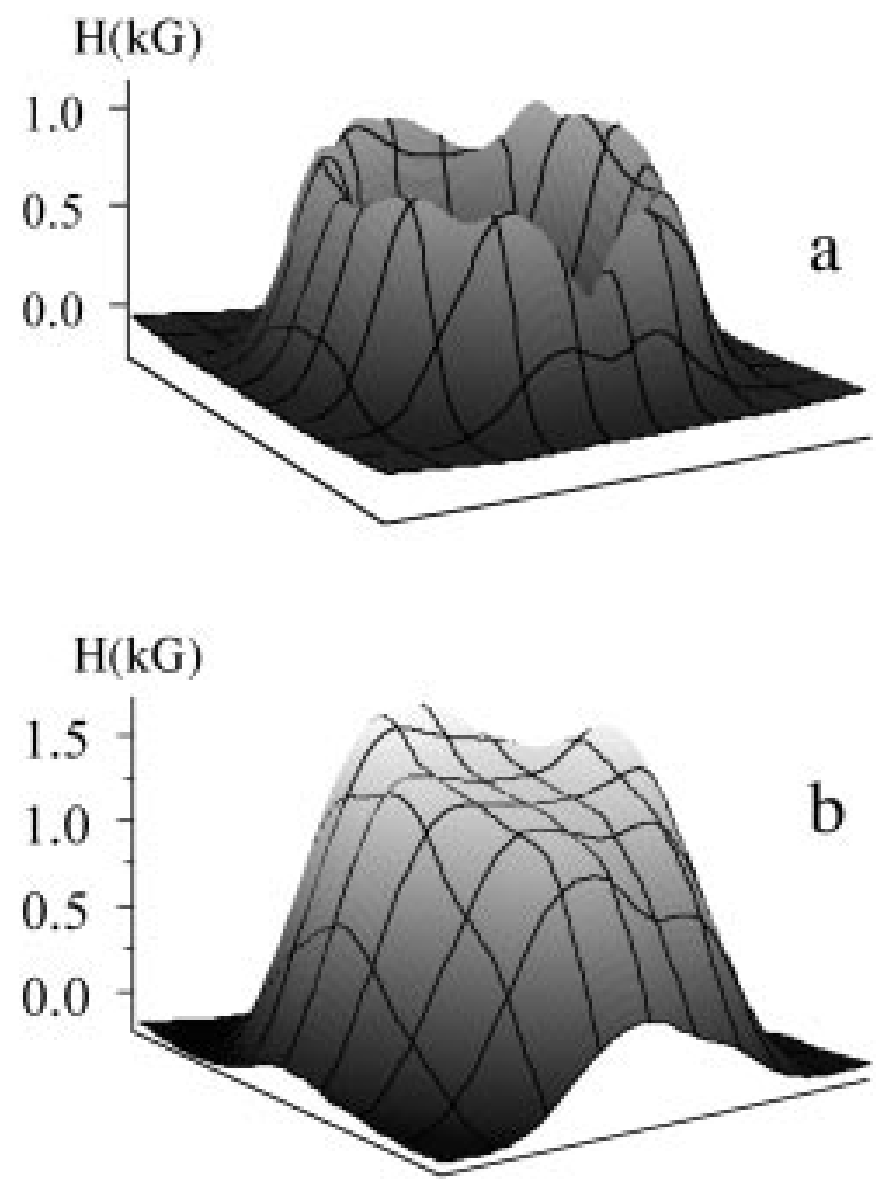

Fig. 5

L. Chen et al. 